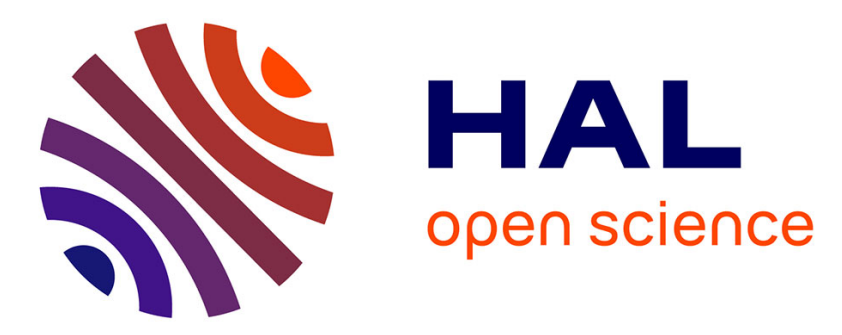

\title{
Nonlinear oscillations of a pendulum cable with the effects of the friction and the radius of the support
}

Charlélie Bertrand, Alireza Ture Savadkoohi, Claude-Henri Lamarque

\section{To cite this version:}

Charlélie Bertrand, Alireza Ture Savadkoohi, Claude-Henri Lamarque. Nonlinear oscillations of a pendulum cable with the effects of the friction and the radius of the support. Nonlinear Dynamics, 2019, 96 (2), pp.1303-1315. 10.1007/s11071-019-04854-5 . hal-02055431v2

\section{HAL Id: hal-02055431 \\ https://hal.science/hal-02055431v2}

Submitted on 14 Jun 2019

HAL is a multi-disciplinary open access archive for the deposit and dissemination of scientific research documents, whether they are published or not. The documents may come from teaching and research institutions in France or abroad, or from public or private research centers.
L'archive ouverte pluridisciplinaire HAL, est destinée au dépôt et à la diffusion de documents scientifiques de niveau recherche, publiés ou non, émanant des établissements d'enseignement et de recherche français ou étrangers, des laboratoires publics ou privés. 


\title{
Nonlinear oscillations of a pendulum cable with the effects of the friction and the radius of the support
}

\author{
C. Bertrand* * A. Ture Savadkoohi . \\ C.-H. Lamarque
}

Received: 11 October 2018 / Accepted: 21 February 2019

\begin{abstract}
The dynamics of a pendular cable with a special constitutive law subjected to a time-dependent velocity is investigated. The Coulomb friction between the cable of pendulum and its support taking into account the effects of the support are considered. To predict system behaviors, the multiple scale method is endowed leading to spot the evolution of the angle and the length of the pendulum. The study permits to enlighten the effects of velocity, friction and the radius of the wheel support on the overall system response and its stability.
\end{abstract}

Keywords Cable pendulum, Varying length, Coulomb friction, method of multiple scales

\section{Introduction}

Cable structures have been used for years in civil engineering structures in a wide range of domain such as bridges or transportation systems. Despite their interest for design, no model is clearly defined to describe every engineering situation. To this purpose, many investigations have been done in cable dynamics but the validity domain of each result is highly related to a specific situation.

Planar behaviors of cables have been investigated by Irvine [1] and their developments are still of practical interests. Some studies considered nonlinear responses of cables under some effects such as temperature [2] or moving loads [3,4]. Other effects for instance aeroelastic provides rich dynamical behavior of cable systems [5].

In the specific domain of transportation system where the cable is subjected to a translating velocity, several approaches have been carried out to compute span modeshapes. One of the first works in this domain, we can name finding modeshapes of a string model subjected to a velocity was carried out by Skutsch [6]. An analytical approach to find the frequencies of mobile elastic cable has been endowed by Simpson in [7] before Triantafyllou [8] traced modal characteristics of translating cables of a single span. These works open to discussion the stability analysis of each span for full telepheric installation. Studies about strings and in the domain of elevators, the response of a translating string subjected to different kinds of boundary conditions has been studied by Gaiko and van Horssen [9].Various studies have already been made in the domain periodic response of varying length pendulum. For example, Belyakov and al. [10] provided analytical and numerical approaches with possible applications to the swing of a swing close to resonance. Other investigationsabout similar system includinganother rheology for the cable have been carried out by Babaz et al. [11] which is adapted in our paper to make further developments about the overall behavior of the system including new consideration about the influence of the radius of the support and induced friction on the behavior of the system, believed to be of practical interest for cable transportation systems. The sophisticated behavior of the sliding process of the cable along a contact surface has been investigated by Betchel et al. in [12] for a torquetransmission system and by Usabiaga et al. in [13] when the sliding of the cable occurs only on a limited portion of the contact interface. Recently, interaction between a static pulley and cable rope model has been investigated through a nodal formulation procedure by Westin and Irani in [14]. Indeed, the authors believe that more investigations should be carried out on the relationship between a pulling velocity, a sophisticated support and the growth of oscillation amplitude. For this reason we propose here a formulation which implies strong interaction between the support and the system in order to re-open the debate on systems subjected to a translating velocity.

Organization of the paper is as it follows: in Sect. (2) the friction considerations are presented, then in Sect. (3) the equations of motions are derived which take into account the effects of friction. Later on in Sect. (4) system equations are treated with the method of multiple scale [15] to predict its response, and to describe the influence of the friction and the radius of the support on the change of amplitude of the oscillations. To illustrate results, Sect. (5) gathers comparisons between analytical predictions and numerical results obtained from numerical time integration to enlighten the pros and the cons of this approach. Then, a representative application to developed techniques in this

C.Bertrand, A. Ture Savadkoohi, C.-H. Lamarque

Univ Lyon, ENTPE, LTDS UMR CNRS 5513, rue Maurice Audin, F-69518 Vaulx-en-Velin Cedex, France

Tel.: +33(0)4 72047246

* Corresponding author: E-mail: charlelie.bertrand@entpe.fr 
paper is provided in Sect. (6) to illustrates the possibilities offered by this resolution. Finally, the paper is concluded in Sect. (7).

\section{System of interest and the friction consideration}

We are looking for the dynamics of a extensible cable wrapped around a pulley with radius $R$. This academic system is depicted in Fig.(1). The mass $m$ is supposed to be concentrated at the end of the cable with initial length and inclination are respectively denoted as $l_{0}$ and $\theta_{0} . T$ is the tension applied on the mass $m$. $\theta$ is the angle between the actual and rest (vertical axis) positions of the pendulum. $l$ is the instantaneous length of the cable. Moreover, the cable is subjected to the velocity $v(t)$ and the wheel is assumed to be undergoing a constant angular velocity $\dot{\omega}$. We will refer respectively to the space or time differentiation of $\bullet$ with $\bullet$ and $\bullet$. To compute accurately the change of

(a)

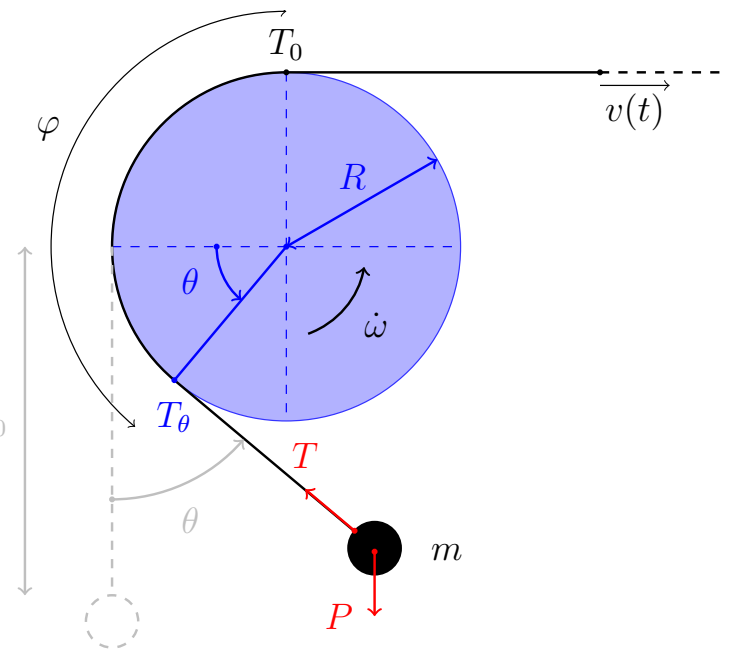

(b)

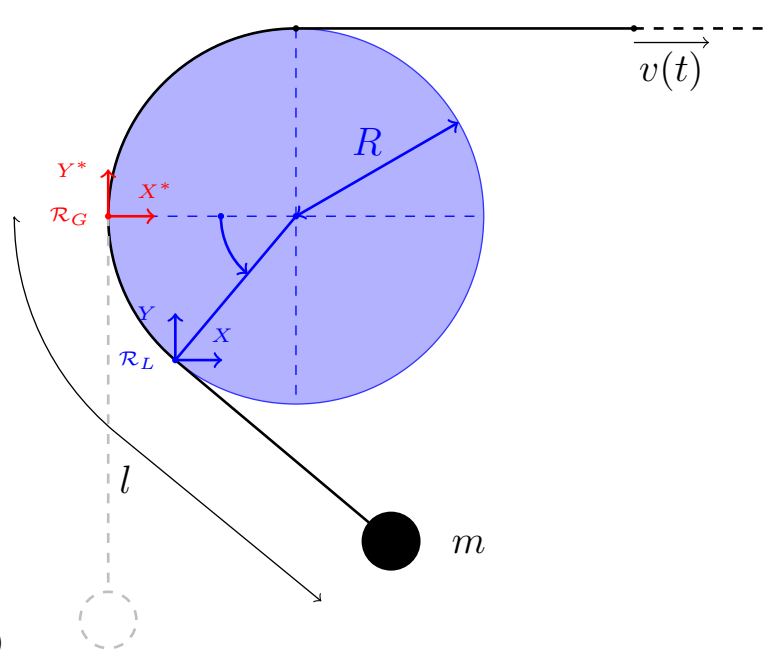

Fig. 1: (a) Varying length pendulum wrapped around a wheel support subjected to velocity and friction - (b) Visualization of the local and global referentials

the length we need the internal elongation variable $x$. The latter allows to express the instantaneous length of the cable as it follows:

$$
l(t)=l_{0}+x(t)-\int_{0}^{t} v(h) \mathrm{d} h
$$

Now, we draw our focus on the tension rheology which we propose as an extension to the formulation given by Babaz et al. in [11]. The latter links the tension at the end of the cable $T$ to the tension at the point the cable leave the contact area that we call it as $T_{\theta}$, see Fig.(1). The rheology is constructed under following considerations:

$$
\mathrm{d} \sigma=E \mathrm{~d} \epsilon
$$

Where $\sigma$ and $\epsilon$ are stress and strain in the cable. Then the tension increment, i.e. $\mathrm{d} T$, along the free-hanging part of the cable is assumed to be linked only to the elongation of the free-hanging part, then it reads:

$$
\begin{aligned}
\mathrm{d} T & =S \mathrm{~d} \sigma \\
& =E S \mathrm{~d} \epsilon \\
& =E S \frac{\mathrm{d}(x-R \theta)}{l-R \theta} \\
\dot{T} & \approx E S \frac{\dot{x}-R \dot{\theta}}{l-R \theta}
\end{aligned}
$$

The approximation described in Eq.(6) holds true only if the strain rate is much smaller than the celerity of the longitudinal waves propagating in the cable (see [11]).

We just derived the time derivative of the tension for the free hanging part of the pendulum, it remains to express the link between the tension imposed at the top of the wheel, $T_{0}$, and the tension at the point where the cable leaves the contact interface, $T_{\theta}$. However in this paper, we assume the simple rheology presented in Eq.(2) taking into account explained assumptions. Then the equilibrium of an infinitesimal cable element in contact with the wheel can be written as a belt friction problem assuming a Coulomb friction associated to $\mu$ coefficient. The intuitive physics underlying this equilibrium are represented in Fig.(2). This reads:

$$
-T^{\prime}(s) \mathrm{d} s \in \mu \operatorname{sgn}\left[v(t)-\dot{\omega}\left(\frac{s}{R}\right) R\right] T(s) \mathrm{d} \varphi
$$




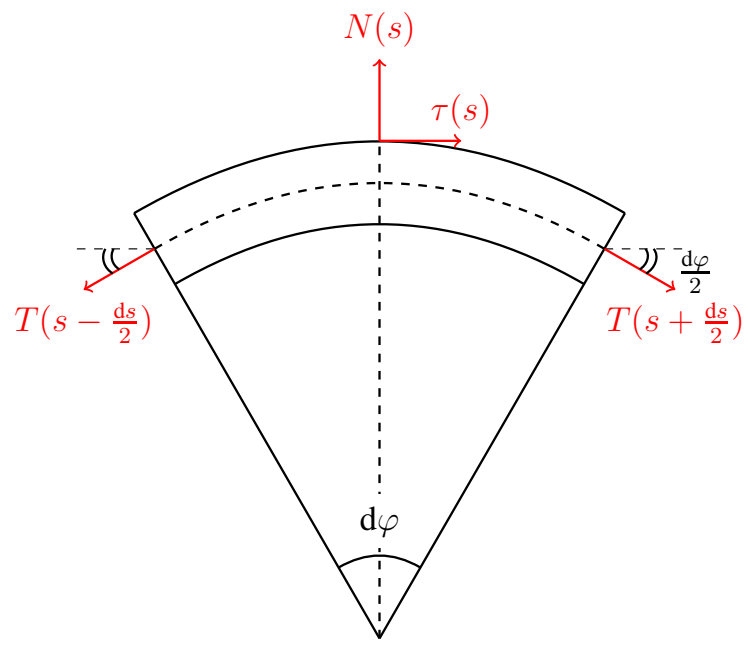

Fig. 2: Concise illustration of the local equilibrium of the cable along the contact interface. $N(s)$ and $\tau(s)$ are normal and tangential components of the tension

Where the notation $\in$ stands for differential inclusion [16]. This approach allows to take into account variation of the reference tension due to the friction and variations of the wrapped angle of the wheel, $\varphi$. Then thanks to geometry, we can express variation of the tension as a function of $\theta$. Since differential inclusion is not practical for analytical treatments, we further assume the case is sliding over the wheel, i.e. $-T^{\prime}(s) \mathrm{d} s= \pm \mu T(s) \mathrm{d} \varphi$. Then this tension variation can be computed as a function of $\theta, \theta_{0}$ et $T_{0}$. Based on this assumption, the cable slides over a segment of the wheel (or wrapped part of the wheel by the cable) which which instantaneous length reads as $R\left(\theta-\theta_{0}\right)$. In another words, once the wheel starts to rotate, the cable begins to (instantaneously) slide with respect to the support. For the rest of the paper, we stay on this assumption about the effect of the friction for further developments. However, formulations involving the full non-linear transition from an initial no-sliding state to a full-sliding one could be essential for a better description of the frictional physics (see e.g. [13]) which are beyond the scope ouf our analysis. As fatigue analysis are beyond the scope of our analysis, a last simplifying assumptions is taken which is a full-sliding configuration which allows to get insight about theoretical worst possible evolution of the tension, then of the amplitude oscillations.

A "positive" friction leads to subsequent developments:

$$
\frac{\mathrm{d} T}{T}=-\mu \mathrm{d} \theta
$$

Then the following can be obtained through integration from the top of the wheel to the point where contact is lost:

$$
T_{\theta}=T_{0} e^{-\mu\left(\theta-\theta_{0}\right)}
$$

Whereas a "negative" friction produces:

$$
T_{\theta}=T_{0} e^{+\mu\left(\theta-\theta_{0}\right)}
$$

Then the time evolution of tensionwhich is presented in Eq.(6) (valid for free hanging part), is modified in order to take into account the effect of the friction based on our assumptions:

$$
\left[\begin{array}{rl}
\dot{T}= & E S \frac{\dot{x}-R \dot{\theta}}{l-R \theta} \mp \dot{\theta} \mu T_{0} e^{\left.\mp \mu\left(\theta-\theta_{0}\right)\right)} \\
& -\quad ; \quad \text { Pulling velocity is bigger than wheel velocity }(v(t)>R \dot{\omega}) \\
& +\quad ; \quad \text { Pulling velocity is smaller than wheel velocity }(v(t)<R \dot{\omega})
\end{array}\right.
$$

It should be mentionned that the Eq.(11) simplifies into the constitutive law given by Babaz et al. in [11] when both friction and wheel radius effects are neglected. It is seen that the friction modifies dynamic variations of the tension around a reference tension. Despite the approximations and assumptions underlying this approach, it opens the path to discussions about fluctuations of tension linked to friction.

\section{Equations of motion}

Due to the wheel support, inertial forces will arise as additional dynamic terms. We write the fundamental principle of dynamics for the mass $m$ in a local referential coordinates. The described dynamics are those offree hanging part 
of the cable pendulum of length $f=l-R \theta$. The location of the mass ( $X$ and $Y$ ) at each instant $t$ yields to:

$$
\left\{\begin{array}{l}
X=f \sin (\theta) \rightarrow \ddot{X}=\ddot{f} \sin (\theta)+2 \dot{f} \dot{\theta} \cos (\theta)+f \ddot{\theta} \cos (\theta)-l \dot{\theta}^{2} \sin (\theta) \\
Y=-f \cos (\theta) \rightarrow \ddot{Y}=-\ddot{f} \cos (\theta)+2 \dot{f} \dot{\theta} \sin (\theta)+f \ddot{\theta} \sin (\theta)+f \dot{\theta}^{2} \cos (\theta)
\end{array}\right.
$$

The local referential $\mathcal{R}_{L}$ is subjected to a varying translation in the global referential $\mathcal{R}_{G}$ (see Fig. (1)):

$$
\left(\begin{array}{l}
X \\
Y
\end{array}\right)_{\mathcal{R}_{L}}=\left(\begin{array}{l}
X^{*} \\
Y^{*}
\end{array}\right)_{\mathcal{R}_{G}}+R\left(\begin{array}{c}
1-\cos (\theta) \\
-\sin (\theta)
\end{array}\right)
$$

Where $X^{*}$ and $Y^{*}$ are fixed references coordinates along the wheel, see Fig.(1). This leads to inertial forces ( $\left.\mathbf{F}_{\mathrm{ie}}\right)$ depending on the dynamics of the system coupled with the wheel support as:

$$
\mathbf{F}_{\mathrm{ie}}=-m R\left(\begin{array}{c}
\ddot{\theta} \sin (\theta)+\dot{\theta}^{2} \cos (\theta) \\
-\ddot{\theta} \cos (\theta)+\dot{\theta}^{2} \sin (\theta)
\end{array}\right)
$$

Then, the equilibrium of the mass $m$ reads:

$$
\left\{\begin{array}{l}
m \ddot{X}=-\sin (\theta) T-m R\left(\ddot{\theta} \sin (\theta)+\dot{\theta}^{2} \cos (\theta)\right) \\
m \ddot{Y}=\cos (\theta) T-m g-m R\left(-\ddot{\theta} \cos (\theta)+\dot{\theta}^{2} \sin (\theta)\right)
\end{array}\right.
$$

From Eqs.(11),(12) and (15), we can obtain:

$$
\begin{aligned}
& \text { If } v_{\text {rel }}>0:\left\{\begin{array}{l}
\ddot{\theta}=-\frac{1}{l-R \theta}(2 i \dot{\theta}+g \sin (\theta))+\frac{R \dot{\theta}^{2}}{l-R \theta} \\
\dddot{l}=-\frac{E S}{m} \frac{\dot{x}-R \dot{\theta}}{l-R \theta}-3(g \sin (\theta)+i \dot{\theta}) \dot{\theta}+R \dot{\theta}^{3}+\dot{\theta} \frac{\mu}{m} T_{0} e^{-\mu\left(\theta-\theta_{0}\right)}
\end{array}\right. \\
& \text { If } v_{\text {rel }}<0:\left\{\begin{array}{l}
\ddot{\theta}=-\frac{1}{l-R \theta}(2 i \dot{\theta}+g \sin (\theta))+\frac{R \dot{\theta}^{2}}{l-R \theta} \\
\dddot{l}=-\frac{E S}{m} \frac{\dot{x}-R \dot{\theta}}{l-R \theta}-3(g \sin (\theta)+i \dot{\theta}) \dot{\theta}+R \dot{\theta}^{3}-\dot{\theta} \frac{\mu}{m} T_{0} e^{+\mu\left(\theta-\theta_{0}\right)}
\end{array}\right.
\end{aligned}
$$

Where the following notation has been set $v_{\text {rel }}=v(t)-\dot{\omega}\left(\frac{s}{R}\right) R$. We clearly see the coupling between $x$ and $\theta$ in Eq. (16). Moreover, neglection of the friction and effects of the radius of the support, i.e. setting $(\mu, R)=(0,0)$, gives consistent model with earlier developments [11]. Our goal is to achieve a prediction of the behavior of the system with the method of multiple scale in order to get insights about the influence of each parameters on system behaviorsincluding worst possible scenarios due to friction effects. The jerk (i.e. term $\dddot{l}$ in Eq. (16)) is interesting to catch growth of the oscillations and to trace a varying tension.

\section{Treating the system with the method of multiple scale}

\subsection{The context}

Let us consider a case where the relative velocity is positive. That is to say that the mass is pulled with velocity $v(t)$ and the friction is opposed to the movement. In order to avoid the integral sign in our further developments, we use the $\mathcal{V}$ to refer to a chosen primitive of $v$. We choose this primitive to be:

$$
\mathcal{V}(t)=\epsilon v_{0} t+\epsilon^{2} a \sin (\Omega t)
$$

Let us define following angular frequencies:

$$
\begin{aligned}
\omega_{1}^{2} & =\frac{g}{l_{0}} \\
\omega_{2}^{2} & =\frac{E S}{m l_{0}} \\
\omega_{0} & =\frac{\omega_{1}}{\omega_{2}}
\end{aligned}
$$

Considering an non-dimensionnalized time $\tau=\omega_{2} t$, the dynamics of the system yields to:

$$
\left\{\begin{aligned}
\ddot{\theta}+\frac{1}{l_{0}+x-\mathcal{V}-R \theta} & \left(l_{0} \frac{\omega_{1}^{2}}{\omega_{2}^{2}} \sin (\theta)+2 \dot{x} \dot{\theta}\right) \\
& -\frac{R \dot{\theta}^{2}}{l_{0}+x-\mathcal{V}-R \theta}=\frac{2 \dot{\mathcal{V}}}{l_{0}+x-\mathcal{V}-R \theta} \dot{\theta} \\
\dddot{x}+l_{0} \frac{\dot{x}-R \dot{\theta}}{l_{0}+x-\mathcal{V}-R \theta} & +3\left(l_{0} \frac{\omega_{1}^{2}}{\omega_{2}^{2}} \sin (\theta)+\dot{x} \dot{\theta}\right) \dot{\theta} \\
& -\dot{\theta} \frac{\mu}{m \omega_{2}^{2}} T_{0} e^{-\mu\left(\theta-\theta_{0}\right)}-R \dot{\theta}^{3}=\left(\dddot{\mathcal{V}}+3 \ddot{\mathcal{V}}^{2}\right)
\end{aligned}\right.
$$


The notation $\bullet$ has been kept for conciseness, i.e. we set $\dot{\bullet}=\frac{\partial}{\partial \tau}$.

A mutiple scale method (MSM) [15] is now endowed to spot the different time responses of the system. We introduce a small parameter $\epsilon \ll 1$ and we define different time scales, fast and slow scales, coupled to each other via $\epsilon$ parameter :

$$
t_{k}=\epsilon^{k} t \quad, \quad k=0,1, \ldots
$$

We can derive new time-differentiation operators $D_{k}=\frac{\partial}{\partial t_{k}}, k=0,1, \ldots$ so [15]:

$$
\begin{aligned}
\frac{\mathrm{d}}{\mathrm{d} t} & =D_{0}+\epsilon D_{1}+\epsilon^{2} D_{2}+\ldots \\
\frac{\mathrm{d}^{2}}{\mathrm{~d} t^{2}} & =D_{0}^{2}+\epsilon\left(2 D_{1} D_{0}\right)+\epsilon^{2}\left(D_{1}^{2}+2 D_{2} D_{0}\right)+\ldots \\
\frac{\mathrm{d}^{3}}{\mathrm{~d} t^{3}} & =D_{0}^{3}+\epsilon\left(3 D_{1} D_{0}^{2}\right)+\epsilon^{2}\left(3 D_{1} D_{0}^{2}+3 D_{2} D_{0}^{2}\right)+\ldots
\end{aligned}
$$

We expand system variables as:

$$
\begin{aligned}
& \theta\left(t_{0}, t_{1}, \ldots\right)=\sum_{n=1}^{3} \epsilon^{n} \theta_{n}\left(t_{0}, t_{1}, \ldots\right)+\mathcal{O}\left(\epsilon^{4}\right) \\
& x\left(t_{0}, t_{1}, \ldots\right)=\sum_{n=2}^{3} \epsilon^{n} x_{n}\left(t_{0}, t_{1}, \ldots\right)+\mathcal{O}\left(\epsilon^{4}\right)
\end{aligned}
$$

and we assume that:

$$
\begin{aligned}
\frac{T_{0}}{m \omega_{2}} e^{\mu \theta_{0}} & =\epsilon T^{*} \\
R & =\epsilon R^{*}
\end{aligned}
$$

In the following section, system equations will be considered at different orders of $\epsilon$ to evaluate temporal responses of system variables.

\subsection{Time responses of the system}

Let us inject Eqs.(21)-(23) in Eq. (19). Governing system equations at different orders of $\epsilon$ read :

$$
\begin{aligned}
& \epsilon^{1}: D_{0}^{2} \theta_{1}+\omega_{0}^{2} \theta_{1}=0 \\
& \epsilon^{2}:\left\{\begin{array}{c}
D_{0}^{2} \theta_{2}+\omega_{0}^{2} \theta_{2}=2 \frac{v_{0}}{l_{0}} D_{0} \theta_{1}-2 \frac{\omega_{0}^{2} v_{0} t_{0}}{l_{0}} \theta_{1}-2 D_{1} D_{0} \theta_{1} \\
D_{0}^{3} x_{2}+D_{0} x_{2}=-a \Omega^{3} \cos \left(\Omega t_{0}\right)-3 \omega_{0}^{2} l_{0} \theta_{1} D_{0} \theta_{1} \\
+\mu T^{*} D_{0} \theta_{1}+R^{*} D_{0} \theta_{1}
\end{array}\right. \\
& \epsilon^{3}:\left\{\begin{aligned}
D_{0}^{2} \theta_{3}+\omega_{0}^{2} \theta_{3}= & -\frac{a \omega_{0}^{2} \sin \left(\Omega t_{0}\right)}{l_{0}} \theta_{1}+\frac{2 a \Omega \cos \left(\Omega t_{0}\right)}{l_{0}} D_{0} \theta_{1}+\frac{\omega_{0}^{2}}{l_{0}} x_{2} \theta_{1} \\
& -\frac{\omega_{0}^{2} v_{0}^{2} t_{0}^{2}}{l_{0}^{2}} \theta_{1}-\frac{\omega_{0}^{2} v_{0} t_{0}}{l_{0}} \theta_{2}+\frac{2 v_{0}^{2} t_{0}}{l_{0}^{2}} D_{0} \theta_{1}+\frac{2 v_{0}}{l_{0}} D_{0} \theta_{2} \\
& -\frac{2}{l_{0}} D_{0} x_{2} D_{0} \theta_{1}-2 D_{2} D_{0} \theta_{1}-2 D_{1} D_{0} \theta_{2}+\frac{2 v_{0}}{l_{0}} D_{1} \theta_{1} \\
& -\frac{\omega_{0}^{2} \theta_{1}^{2}}{l_{0}} R^{*}+\frac{\left(D_{0} \theta_{1}\right)^{2}}{l_{0}} R^{*}+\frac{\omega_{0}^{2}}{6} \theta_{1}^{3}-D_{1}^{2} \theta_{1} \\
D_{0}^{3} x_{3}+D_{0} x_{3}= & \frac{v_{0} t_{0}}{l_{0}}\left(R^{*} D_{0} \theta_{1}-D_{0} x_{2}\right)-3 D_{1} D_{0}^{2} x_{2}-D_{1} x_{2} \\
& -3 \omega_{0}^{2} l_{0} \theta_{2} D_{0} \theta_{1}-3 \omega_{0}^{2} l_{0} \theta_{1}\left(D_{1} \theta_{1}+D_{0} \theta_{2}\right) \\
& -\mu T^{*}\left[\mu \theta_{1} D_{0} \theta_{1}-D_{1} \theta_{1}-D_{0} \theta_{2}\right] \\
& +3 v_{0}\left(D_{0} \theta_{1}\right)^{2}+R^{*}\left(D_{1} \theta_{1}+D_{0} \theta_{2}\right)
\end{aligned}\right.
\end{aligned}
$$

Order $\epsilon^{3}$ is sufficient to predict overall evolution trend of the system and to get insight about the way the parameters of the system influence the amplitude of each oscillation. We see that every time response can be obtained as the 
solution of an harmonic oscillator with a non zero right hand side, except for the fast time response which corresponds to a purely harmonic behavior. Resonant terms lead the system to unbounded solutions. However, we aim to catch the behavior of the system for finiteand local durations [15]. In this particular case, secular terms are not removedin order to get insight about parameters that would leads the systems to unbounded growth.Note that we do not seek for a periodic solution in this case, indeed the system is only valid for a finite duration of time since the mass can be physically pulled out of the pulley when $t$ becomes too big. Moreover the pulling velocity injects intrinsic divergence to the system, that makes the inference of the influence of every parameter, including friction, very challenging. The goal is to predict the overall behavior of the system and to measure the effects of each physics on the growth of the unknowns $\theta$ and $x$.

Every solutions can be written as:

$$
\begin{aligned}
& \theta_{k}=\Theta_{k, 1} \cos \left(\omega_{0} t_{0}\right)+\Theta_{k, 2} \sin \left(\omega_{0} t_{0}\right)+\sum_{l=0}^{N}\left(f_{\theta_{k}, l}-\mu g_{\theta_{k}, l}\left(\mu, R^{*}\right)\right) t_{0}^{l} \\
& x_{k}=\xi_{k, 1} \cos \left(t_{0}\right)+\xi_{k, 2} \sin \left(t_{0}\right)+\xi_{k, 3}+\sum_{l=0}^{N}\left(f_{x_{k}, l}-\mu g_{x_{k}, l}\left(\mu, R^{*}\right)\right) t_{0}^{l}
\end{aligned}
$$

$f_{\theta_{k}, i}$ refers to a coefficient which is not depending on the $T^{*}$ and $R^{*}$ coefficients, i.e. it is not function of the friction and the radius of the support. On the contrary $g_{\theta_{k}, i}\left(\mu, R^{*}\right)$ refers to a coefficient which is only depending on the $T^{*}$ and $R^{*}$ coefficients. The presented solutions in Eq. (27) and Eq. (28) refer to a very general case, imposing very long expressions. In the next section, we treat a particular case, showing some application of these results.

\section{Comparisons for a special situation}

\subsection{Analytical application}

We consider a flawless pull of the mass so that $a=0$ and that the mass is inclined with $\theta=\theta_{0}$ at the initial time. The mass is released without any velocity nor acceleration. As a summary, we consider following initial conditions relevant to elongation, $x(t)$, and the angle, $\theta(t)$ :

$$
t=0 \rightarrow(\ddot{x}(0), \dot{x}(0), x(0), \dot{\theta}(0), \theta(0))=\left(0,0,0,0, \theta_{0}\right)
$$

We can evaluate every time response in this particular case. We choose the first non zero time responses to be carrying the initial condition. After developments, components of Eq. (27) and Eq. (28) can be obtained (see Eq. (34) and (35) in App. (A) for detailed expressions).

From Eq. (34) and (35), it is seen that the friction (via $\mu$ parameter) and the support (via $R^{*}$ parameter), influence the system behavior. As a consequence, neglecting those phenomena can be carried out by setting $\mu=0$ and $\tilde{R}=0$. When injected in the general solutions, the time responses read as:

$$
\left\{\begin{aligned}
\theta_{1}\left(t_{0}\right)= & \theta_{0} \cos \left(\omega_{0} t_{0}\right) \\
\theta_{2}\left(t_{0}\right)= & -\frac{3 \theta_{0} v_{0} \sin \left(\omega_{0} t_{0}\right)}{4 \omega_{0} l_{0}}-\frac{\theta_{0} \omega_{0} v_{0} \sin \left(\omega_{0} t_{0}\right)}{4 l_{0}} t_{0}^{2}+\frac{3 \theta_{0} v_{0} \cos \left(\omega_{0} t_{0}\right)}{4 l_{0}} t_{0} \\
\theta_{3}\left(t_{0}\right)= & -\frac{\theta_{0}^{3}\left(1152\left(\omega_{0}^{2}-1\right) \omega_{0}^{5} \sin \left(t_{0}\right) \sin \left(\omega_{0} t_{0}\right)+\left(196 \omega_{0}^{4}-53 \omega_{0}^{2}+1\right) \cos \left(3 \omega_{0} t_{0}\right)\right)}{192\left(1-4 \omega_{0}^{2}\right)^{2}} \\
& +\frac{\theta_{0}^{3}\left(1728 \omega_{0}^{6} \cos \left(t_{0}\right)-1728 \omega_{0}^{6}+196 \omega_{0}^{4}-53 \omega_{0}^{2}+1\right) \cos \left(\omega_{0} t_{0}\right)}{192\left(1-4 \omega_{0}^{2}\right)^{2}} \\
& +\mu \frac{\theta_{0}^{2} T^{*}\left(\left(-3 \omega_{0}^{4} \cos \left(t_{0}\right)+3 \omega_{0}^{4}-4 \omega_{0}^{2}+1\right) \cos \left(\omega_{0} t_{0}\right)\right)}{\left(\omega_{0}-1\right)\left(\omega_{0}+1\right)\left(2 \omega_{0}-1\right)\left(2 \omega_{0}+1\right) l_{0}} \\
& +\mu \frac{\theta_{0}^{2} T^{*}\left(\left(4 \omega_{0}^{2}-1\right) \cos ^{2}\left(\omega_{0} t_{0}\right)+2\left(\omega_{0}^{2}-1\right) \omega_{0}^{3} \sin \left(t_{0}\right) \sin \left(\omega_{0} t_{0}\right)\right)}{\left(\omega_{0}-1\right)\left(\omega_{0}+1\right)\left(2 \omega_{0}-1\right)\left(2 \omega_{0}+1\right) l_{0}} \\
& -\frac{\theta_{0} \omega_{0}^{2} v_{0}^{2} \cos \left(\omega_{0} t_{0}\right)}{32 l_{0}^{2}} t_{0}^{4}-\frac{5 \theta_{0} \omega_{0} v_{0}^{2} \sin \left(\omega_{0} t_{0}\right)}{16 l_{0}^{2}} t_{0}^{3}+\frac{15 \theta_{0} v_{0}^{2} \cos \left(\omega_{0} t_{0}\right)}{32 l_{0}^{2}} t_{0}^{2} \\
& +c_{1}\left(t_{0}\right) R^{*}+c_{2}\left(t_{0}\right) t_{0}
\end{aligned}\right.
$$




\begin{tabular}{|r|r|r|r|r|r|r|r|}
\hline$l_{0}(\mathrm{~m})$ & $g\left(\mathrm{~m} . \mathrm{s}^{-2}\right)$ & $E S(\mathrm{GN})$ & $m(\mathrm{~kg})$ & $T_{0}(\mathrm{kN})$ & $v_{0}\left(\mathrm{~m} . \mathrm{s}^{-1}\right)$ & $R(\mathrm{~m})$ & $\mu$ \\
\hline 100 & 9.81 & 108 & 1000 & 8.495 & 1 & 2 & 0.4 \\
\hline
\end{tabular}

Table 1: System parameters

\begin{tabular}{|r|r|r|r|}
\hline$\omega_{1}\left(\mathrm{rad} . \mathrm{s}^{-1}\right)$ & $f_{1}\left(\mathrm{~s}^{-1}\right)$ & $\omega_{2}\left(\mathrm{rad} . \mathrm{s}^{-1}\right)$ & $f_{2}\left(\mathrm{~s}^{-1}\right)$ \\
\hline 0.313 & 0.049 & 32.7 & 5.21 \\
\hline
\end{tabular}

Table 2: System frequencies

$$
\left\{\begin{aligned}
x_{2}\left(t_{0}\right)= & -\frac{3 \theta_{0}^{2} l_{0}\left(\omega_{0}^{2}-1\right) \omega_{0}^{2}}{4\left(4 \omega_{0}^{4}-5 \omega_{0}^{2}+1\right)}\left(4 \omega_{0}^{2} \cos \left(t_{0}\right)-\cos \left(2 \omega_{0} t_{0}\right)-4 \omega_{0}^{2}+1\right) \\
& -\frac{\theta_{0}\left(4 \omega_{0}^{2}-1\right)\left(R^{*}+\mu T^{*}\right)}{\left(4 \omega_{0}^{4}-5 \omega_{0}^{2}+1\right)}\left(-\omega_{0}^{2} \cos \left(t_{0}\right)+\cos \left(\omega_{0} t_{0}\right)+\omega_{0}^{2}-1\right) \\
x_{3}\left(t_{0}\right)= & c_{3}\left(t_{0}\right) t_{0}^{2}-\frac{15 \theta_{0}^{2} v_{0} \omega_{0}^{3}\left(\omega_{0}\left(4 \omega_{0}^{2}-3\right) \sin \left(t_{0}\right)+\sin \left(2 \omega_{0} t_{0}\right)\right)}{4\left(1-4 \omega_{0}^{2}\right)^{2}} \\
& +\frac{1}{8} \theta_{0} v_{0}\left(\frac{2\left(\mu T^{*}-3 R^{*}\right) \cos \left(\omega_{0} t_{0}\right)-6 \omega_{0}^{2} \cos \left(t_{0}\right)\left(R^{*}+\mu T^{*}\right)}{l_{0}\left(\omega_{0}^{2}-1\right)}\right) t_{0} \\
& +\frac{1}{8} \theta_{0} v_{0}\left(\frac{3 \theta_{0} \omega_{0}^{2}\left(6 \omega_{0}^{2} \cos \left(t_{0}\right)+\cos \left(2 \omega_{0} t_{0}\right)+4 \omega_{0}^{2}-1\right)}{4 \omega_{0}^{2}-1}\right) t_{0} \\
& +\frac{\theta_{0}^{2} \mu^{2} T^{*}\left(-4 \omega_{0}^{2} \cos \left(t_{0}\right)+\cos \left(2 \omega_{0} t_{0}\right)+4 \omega_{0}^{2}-1\right)}{16 \omega_{0}^{2}-4} \\
& +\frac{\theta_{0} v_{0} \omega_{0}^{2} \sin \left(t_{0}\right)\left(3 R^{*}\left(\omega_{0}^{2}-5\right)+\mu T^{*}\left(3 \omega_{0}^{2}-7\right)\right)}{4 l_{0}\left(\omega_{0}^{2}-1\right)^{2}} \\
& +\frac{\theta_{0} v_{0} \sin \left(\omega_{0} t_{0}\right)\left(3 R^{*}\left(5 \omega_{0}^{2}-1\right)+\mu T^{*}\left(3 \omega_{0}^{2}+1\right)\right)}{4 l_{0} \omega_{0}\left(\omega_{0}^{2}-1\right)^{2}} \\
&
\end{aligned}\right.
$$

where $c_{1}, c_{2}$ and $c_{3}$ are scalar functions detailed in App. (A).

We see that the friction has an effect on the unbounded responses of the system, however the friction and the radius (contact area) are too small in reality to play enormous roles in reducing the growth of the oscillations. From Eq. (30) and (31), we notice that the amplitude of every components of the motion are functions of the initial angle, $\theta_{0}$. We also see that intrinsic diverging terms arise at slow time scales for $\theta$ as a direct consequence of the pulling velocity and the initial angle. Also the divergence is spotted to be quadratic in time during slow time scale. From these observations, effect of the friction/support can not be used for control purposes because we cannot change these parameters for each engineering situations. From Eq.(30) it can be seen that the $\theta$ is oscillating with two frequencies namely 1 and $\omega_{0}$ which are linked to the cable and the pendulum frequencies, respectively. Equation (31) illustrates that the elongation of the cable, in addition to the frequencies 1 and $\omega_{0}$, is carried out with frequency $2 \omega_{0}$ (see Eq.(18)). However, the frequency $\omega_{0}$ is always linked to the effects of the friction and the radius of the wheel.

However, the effect of the friction and the wheel on elongation (and then tension) seems to be way more visible. Our study also enlights the major role played by the effects of the support in the elongation amplitude compared to the friction. Indeed, these phenomena are seen not to be comparable because of the difference between the dimension of the radius for the wheel and induced elongation due to the effect of the friction.

\subsection{Numerical comparisons}

Here, results obtained via the direct numerical integration of the system equations Eq. (19) are compared with these which are obtained via MSM as defined in Eq. (30) and Eq. (31). Let us consider the system parameters collected in Tab. (1). System frequencies obtained from Eqs.(18) are collected in Tab.(2). Time integration is done over a time period of $35 \mathrm{~s}$ via the ode 45 function of Matlab ${ }^{\circledR}$ with following criteria:

$$
\begin{aligned}
& h=7.5 \times 10^{-4} ; \quad \text { (time step) } \\
& \text { RelTol }=10^{-14} \\
& \text { AbsTol }=10^{-14} \\
& \text { NormControl }=10^{-14}
\end{aligned}
$$


We plot simultaneously every variables over a time interval of $35 \mathrm{~s}$ with a chosen $\epsilon=2 \%$. The choice of $\epsilon$ permits to evaluate the rescaled parameters $T^{*}$ and $R^{*}$ which are directly related to physics of the system (see Eq.(23)). Note that choosing $\epsilon$ parameter value is doable since it allows to compute rescaled parameters and check the compatibility of designed system parameters for the time period studied. Further considerations about the precision of such methodologies are discussed in [17]. Moreover, the choice of $\epsilon$ parameter permits to have an idea about "starts" of the infinity of each time scale, as shown by Lamarque et al. [18]. This leads to stay within the time $t \in\left[0, \mathcal{O}\left(\frac{1}{\epsilon}\right)\right]$ for being sure that we cover at least the transient response. The overall trend of the motion is well restituted by obtained results with MSM. Obtained results with MSM and those obtained via direct numerical integration of the system equations and depicted in Figs.(3)-(5). Moreover, we see that the observations done about frequencies (see Tab.(2) are in good agreement with numerical results. Indeed, the change of instantaneous frequency due to the dynamics and the varying length are not caught precisely when the considered time $t$ is too big. Eventually, the amplitude of the prediction stays acceptable only for small duration. This can be explained by the unbounded growth of some motion components and that our solutions consist of successive approximations. Referring to Figs.(3)-(5), the reader may observe that the growth of oscillations, friction induced elongations and overall instantaneous frequency is well approximated under our assumptions and developments. The trajectory of the mass and the tension can also be derived analytically from

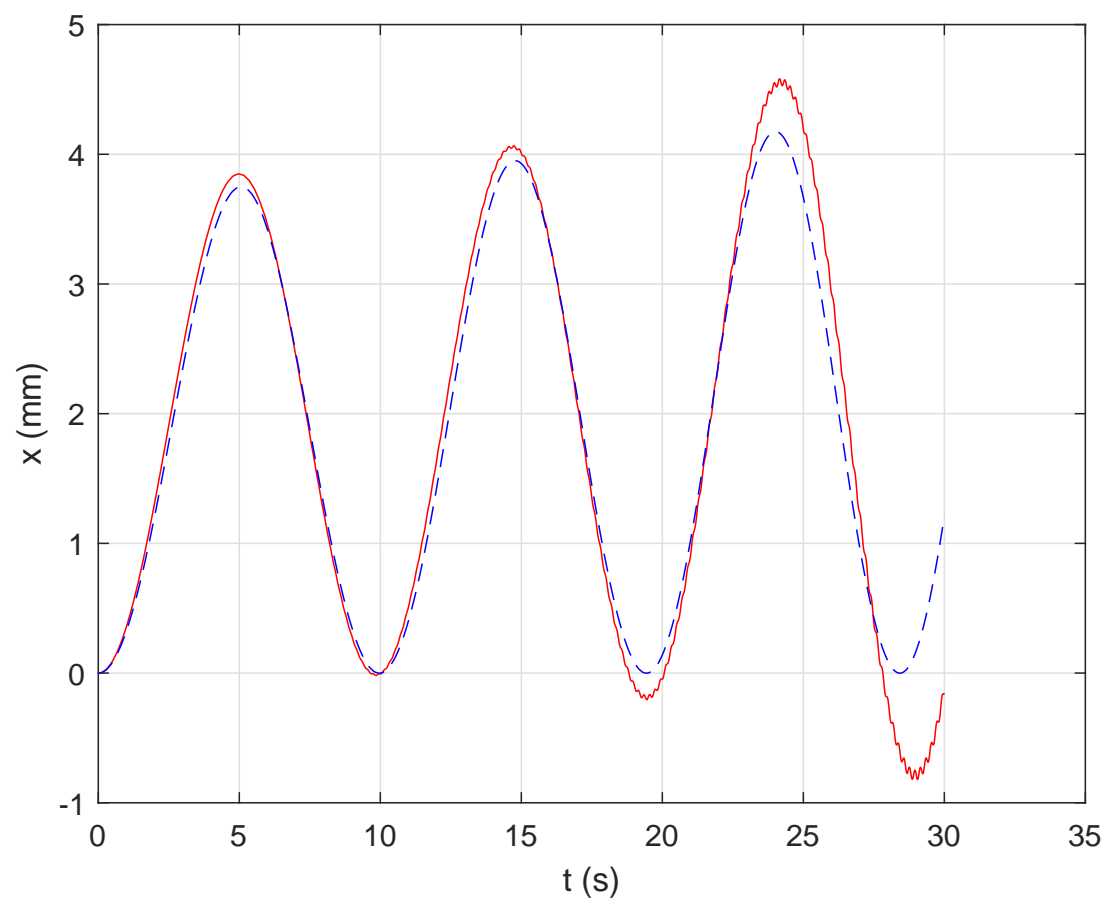

Fig. 3: Time histories of the elongation $x$; (solid line - - ), obtained from Eq. (31); (dashed line - - -) evaluated from numerical integration of Eq. (19). The effects of friction and the radius of the support are ignored i.e. $(\mu, R)=(0,0)$

Eqs.(30) and (31) to give insights about the influence of friction and support on the motion. Figures (7) and (8) show the tajectories obtained from direct numerical integration of Eq. (19). As analytical predictions were very close to numerical ones until $t=35 \mathrm{~s}$, we did not include them in figures. We present the plots for the time integration also to validate our assumptions on tension change due to friction and the influence of the wheel. Figure (8) shows that the relative difference between tensions at final step, for the systems with and without inclusion of the effect of friction, $\Delta T$ is very small providing $\frac{\Delta T}{T_{0}} \approx 10^{-3}$ so that putting the effect of the friction at order $\epsilon$ for analytical developments was relevant (see Eqs.(23)). Looking at Figs.(4), (6) and (8) we see that the radius of the support plays an important role in the dynamics of the overall system while the effect of the friction (in the limits of its physical parameters [19]) can be neglected.

The frequency content variations of the system and also the main trend of frequency can also be caught via the Hilbert transform. Within the range of engineering parameters, frequency consists of variations around a main frequency as depicted in Figs.(9)-(12). The same behavior can be spotted with MSM and can be compared with the signal obtained from time integration. These figures show that the traced instantaneous frequencies obtained from the results of numerical integration of system equations are in reasonable agreements with those which are obtained from analytical developments, see Eqs.(30) and (31) (the error is at the order of $\epsilon$ ). 


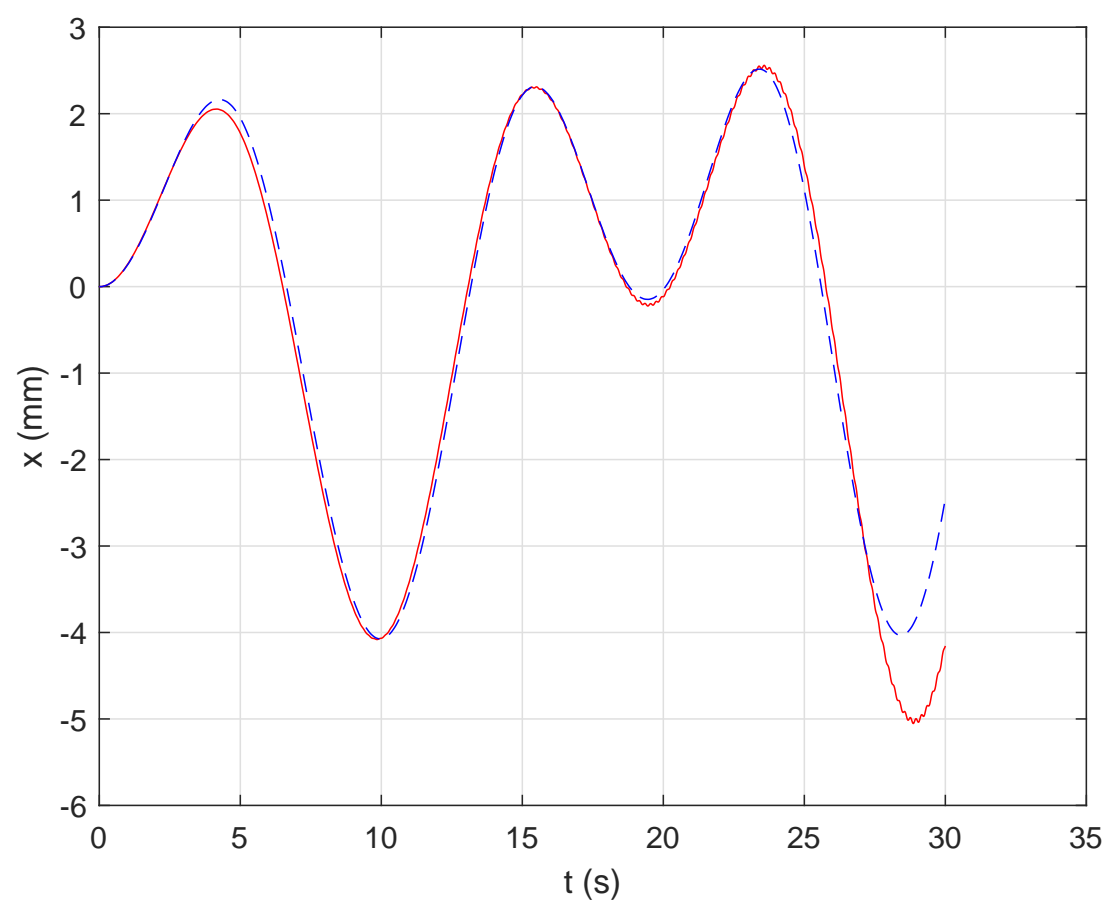

Fig. 4: Time histories of the elongation $x$; (solid line - ), obtained from Eq. (31); (dashed line - - -) evaluated from numerical integration of Eq. (19) with the friction coefficient $\mu>0$ and ignoring the effects of the radius of the support $R=0$

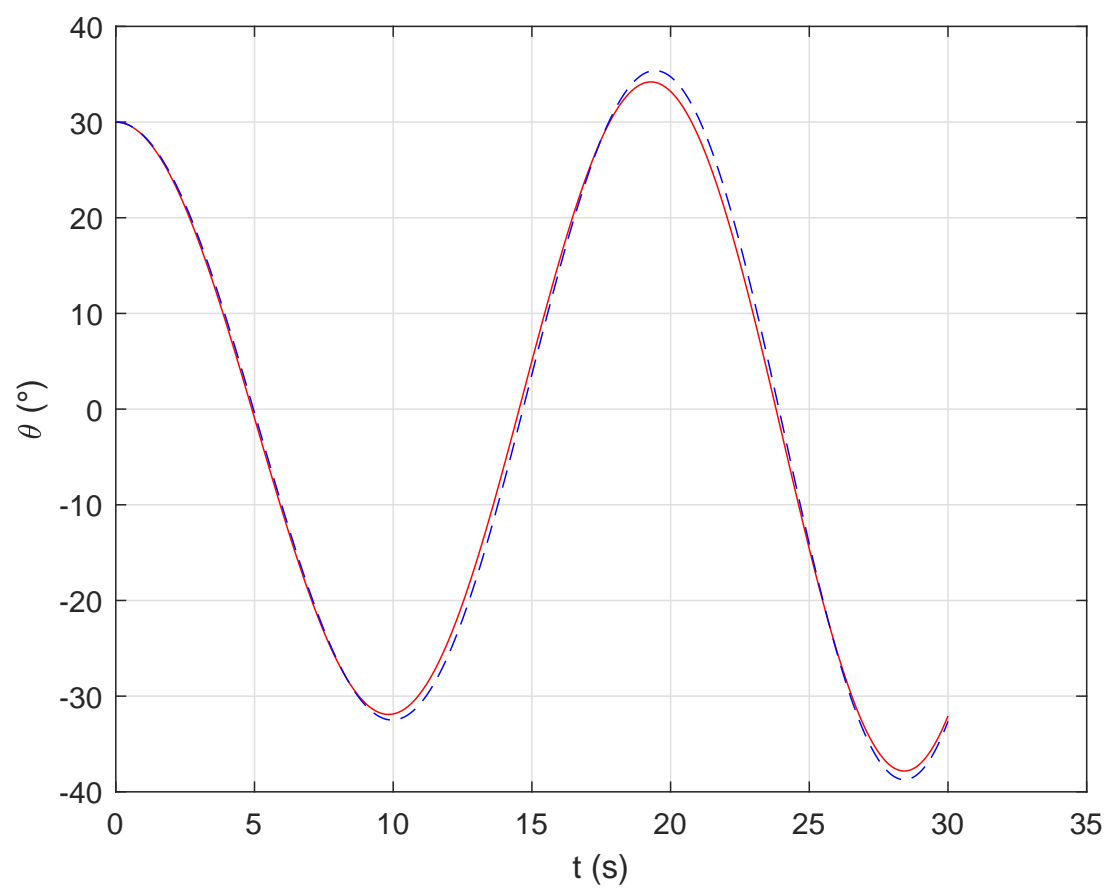

Fig. 5: Time histories of the angle $\theta$; (solid line - ), obtained from Eq. (30); (dashed line - - -) evaluated from numerical integration of Eq. (19). The effects of friction and the radius of the support are ignored i.e. $(\mu, R)=(0,0)$

\section{An application}

Let us consider a situation where a drive pulley is used to pull a mass $m$ out of an encased space. The considered system is depicted in Fig.(13). The mass should not hit the surrounding barriers located at a distance $d$ from the its rest position. We denote the limit angle which causes the mass to hit the wall as $\theta_{d}$. The methodology consists of finding the roots of $|X(t)|-d$, where the time $t$ corresponds to the real physical time:

$$
X(t)=\left(l_{0}+x(t)-v_{0} t-R \theta(t)\right) \sin (\theta(t))-R(1-\cos (\theta(t)))
$$

$\theta(t)$ and $x(t)$ can be obtained from Eqs.(30) and (31) where the rescaled time $t_{0}$ is set to $\omega_{2} t$.

If numerical roots can be found within the range $t \in\left[0, \frac{l_{0}}{v_{0}}\right]$, then the surrounding barriers will not be touched by 


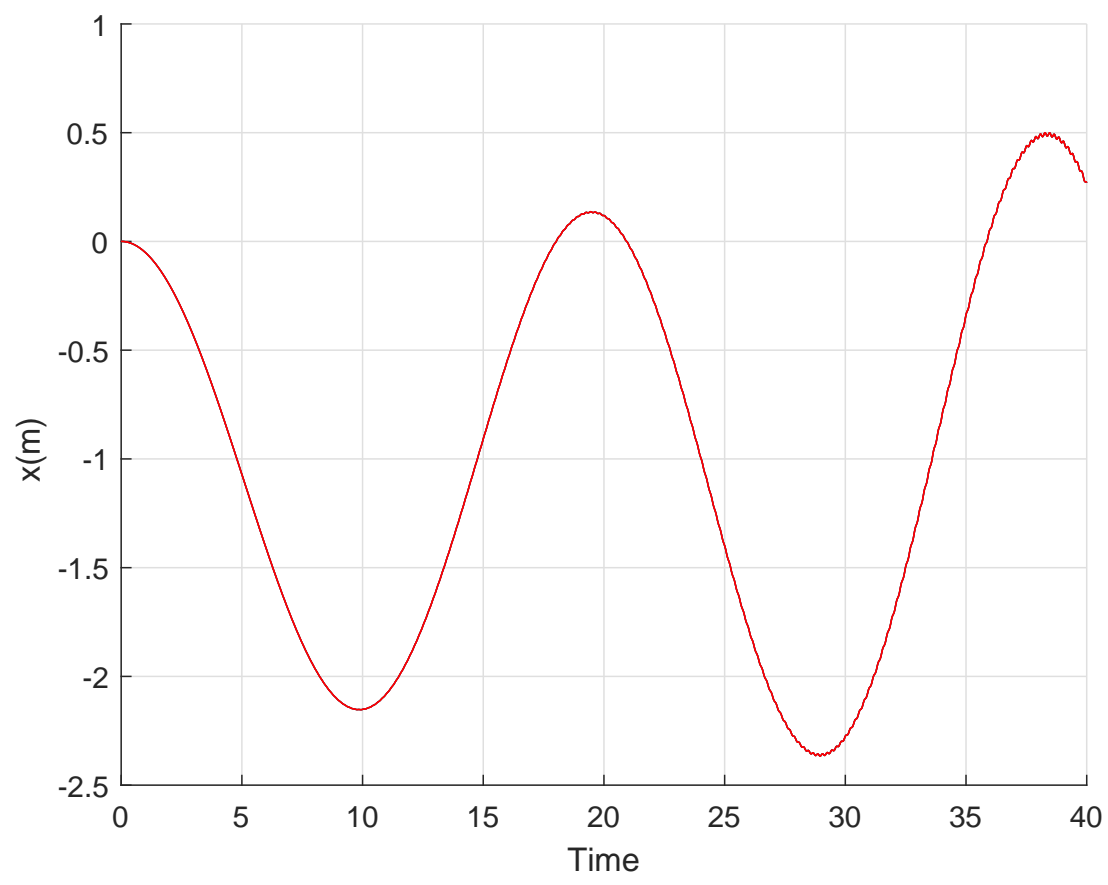

Fig. 6: Analyticaly obtained results for elongation $x$ (solid line - - ), obtained from Eq. (31) ignoring the effect of the friction $(\mu=0)$ and considerating the effects of the wheel $(R=2 \mathrm{~m})$

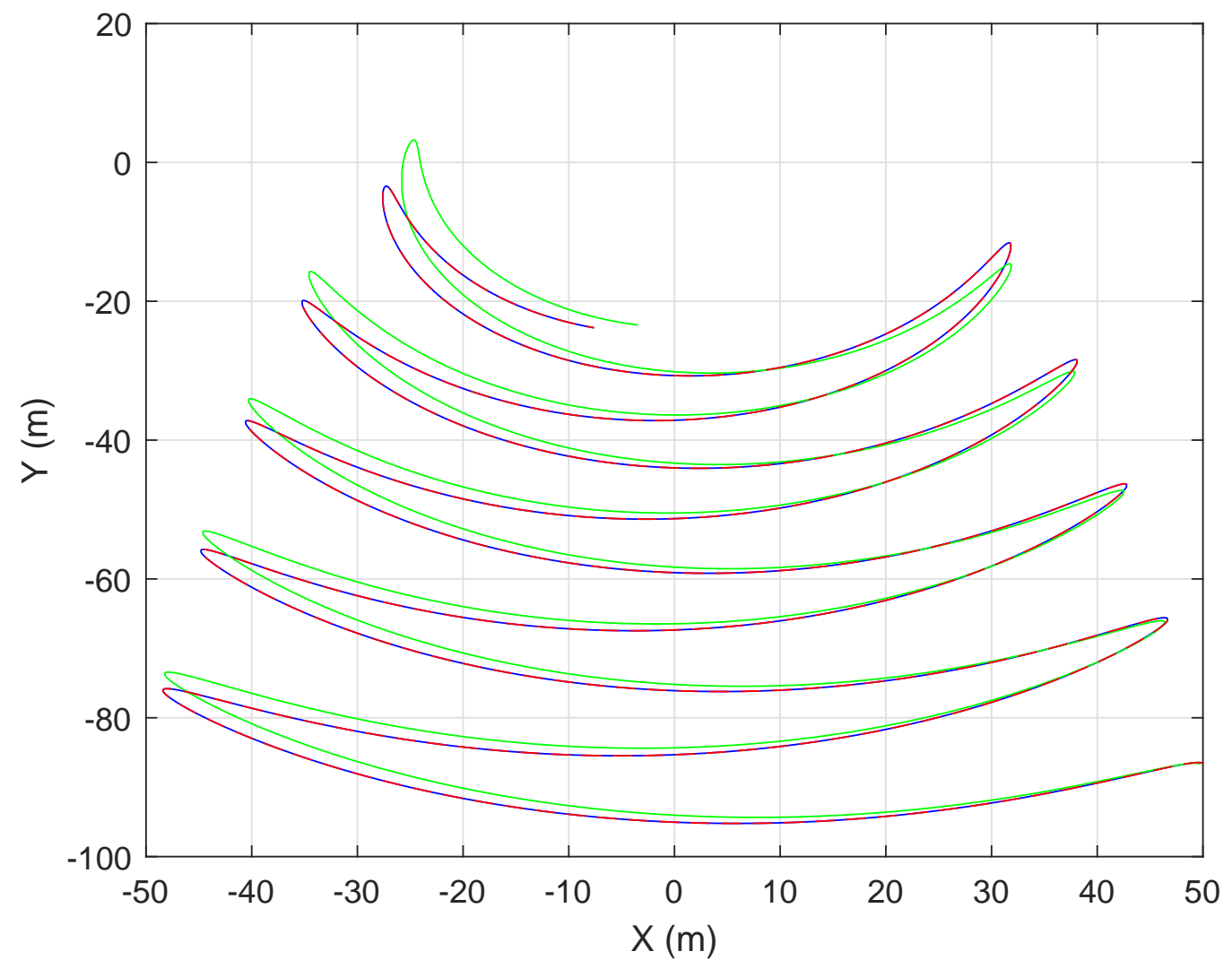

Fig. 7: Mass trajectory for different friction and support parameters: $\mu=0, R=0$ (dashed line - - -) ; $\mu=0.4$, $R=0$ (solid line - ) $; \mu=0.4, R=2 \mathrm{~m}$ (solid line - ). Results are obtained from direct numerical integration of Eq. (19)

the mass. Due to the competition between $\omega_{1}$ and $\omega_{2}$, several behaviors for the system are expected. As numerical examples, we consider two cases with different system parameters which are collected in Tab.(3). It is seen that the system of the case (1) never hits the barriers while the system of the case (2) hits the barrier. Moreover, the impact time can be obtained numerically from Eq.(33) which is presented in Fig.(14). 


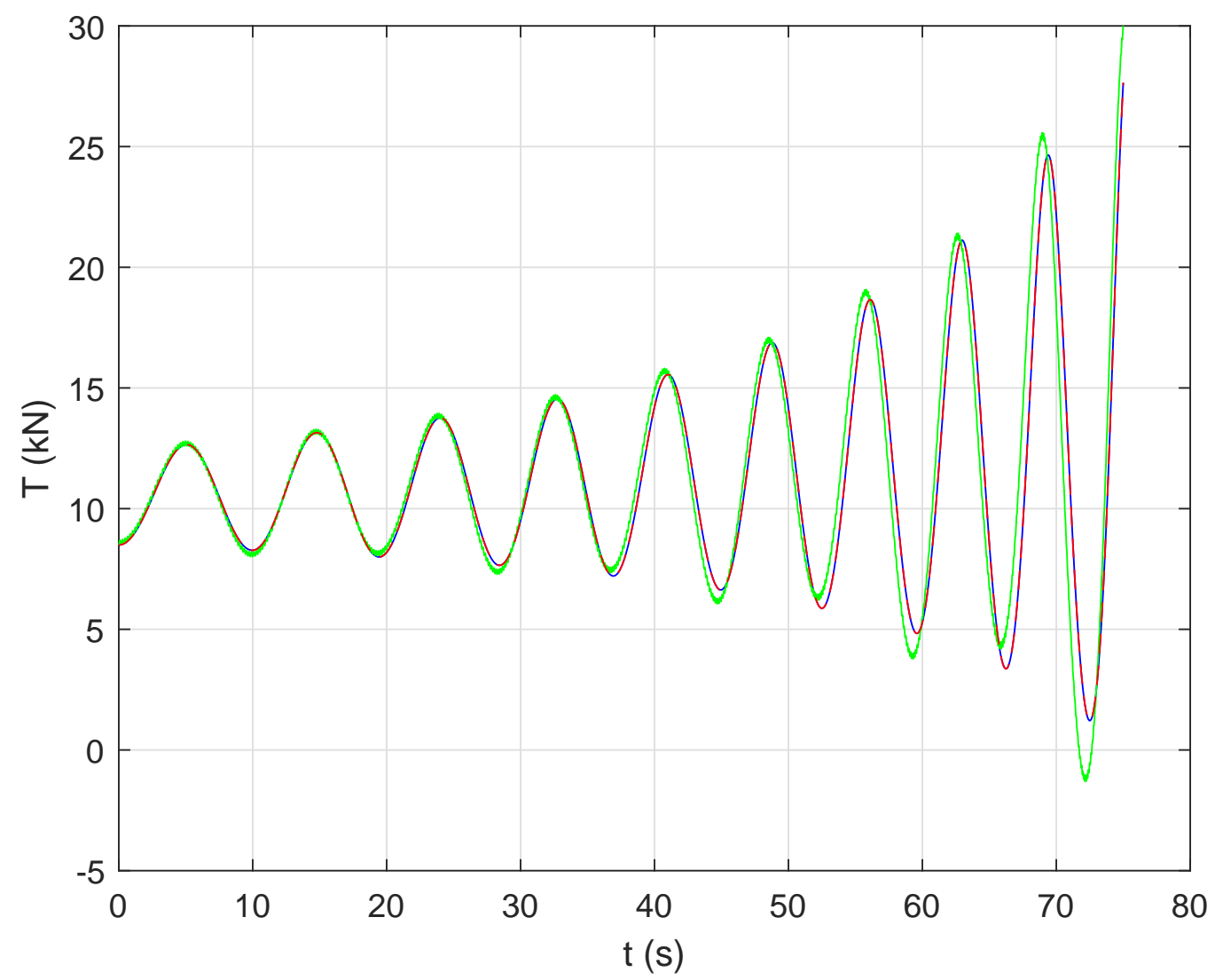

Fig. 8: Tension for different friction and support parameters: $\mu=0, R=0$ (dashed line - - -) ; $\mu=0.4, R=0$ (solid line - ) ; $\mu=0.4, R=2 \mathrm{~m}$ (solid line - ) Results are obtained from direct numerical integration of Eq. (19)

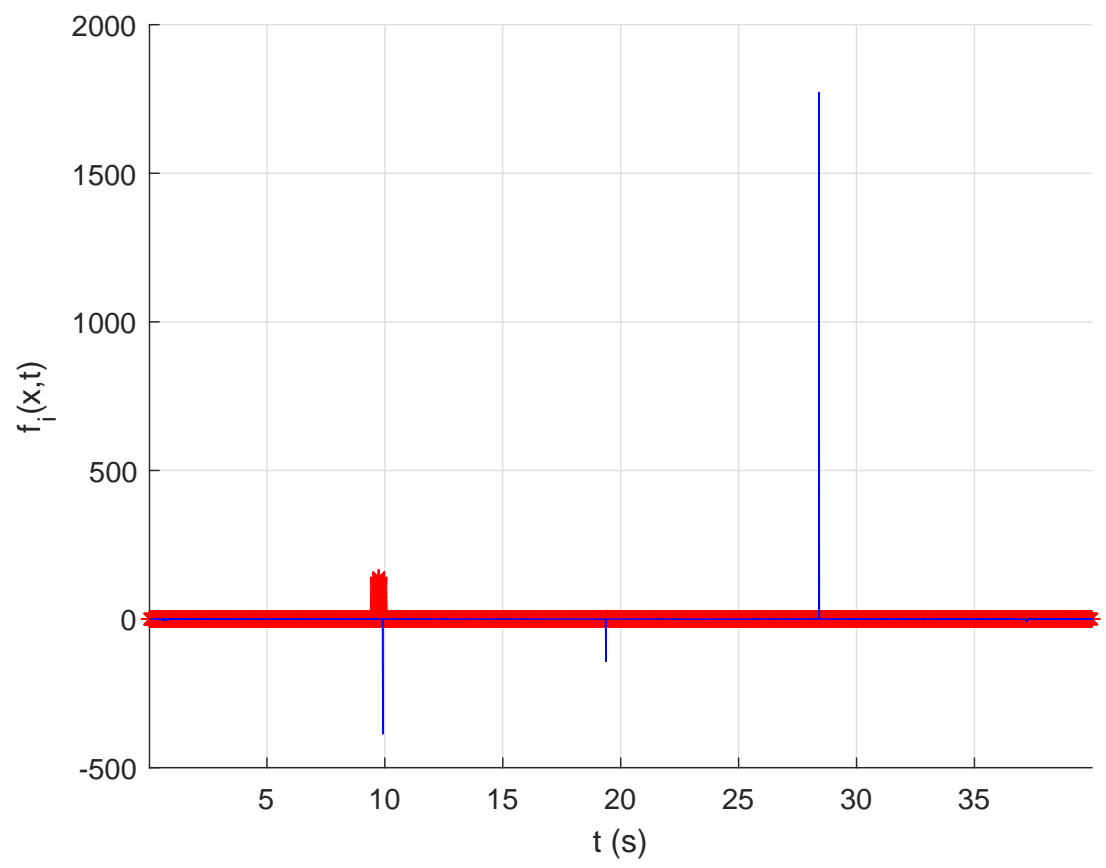

Fig. 9: Instantaneous frequency of $x(t)$ obtained from analytical prediction (see Eq. (31) (solid line - ) and from direct integration of Eq. (19) (solid line -) for $t \in[0,35] \mathrm{s}$

\section{Conclusions}

Nonlinear oscillations of a cable pendulum with a special rheology subjected to a time dependent velocity taking into account the effects of the radius of the wheel support and the induced friction is studied. Governing system equations are treated by the method of multiple scale without eliminating the diverging terms. It is found that the radius of the support and the induced friction affect system behaviors. However, the influence of the wheel is deeper with respect to 


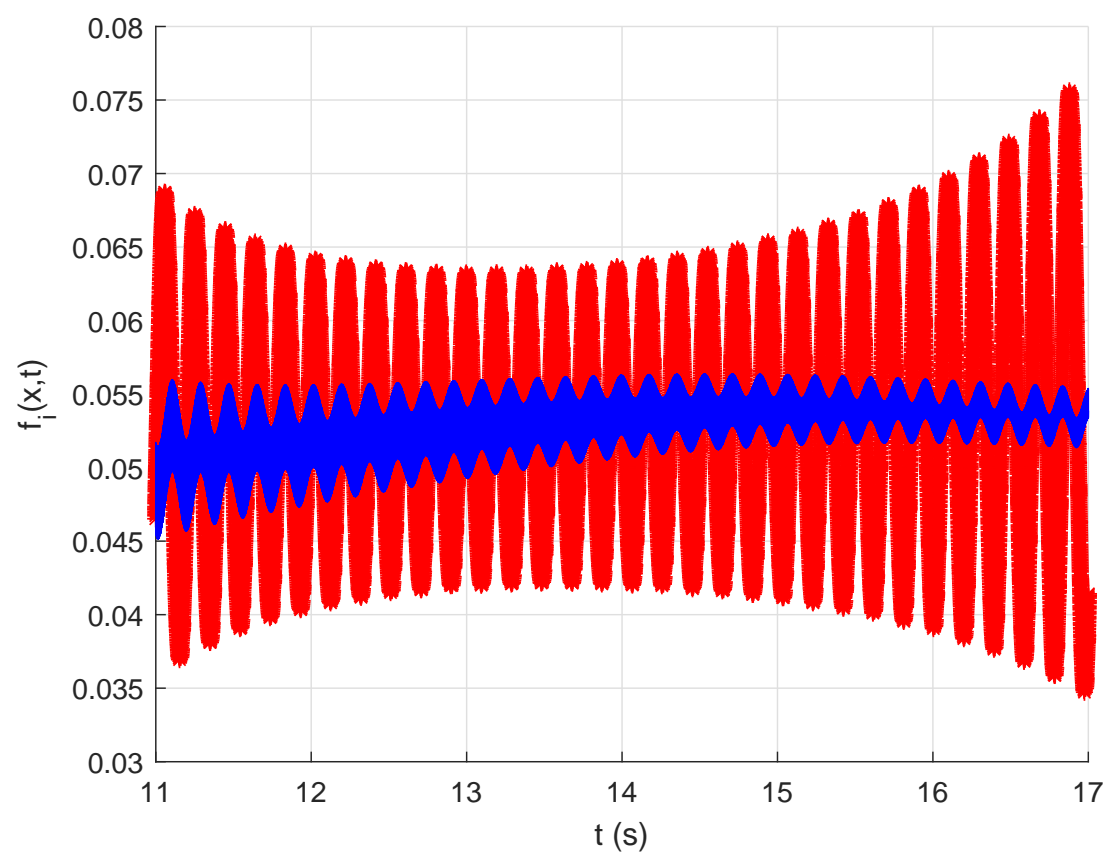

Fig. 10: Instantaneous frequency of $x(t)$ obtained from analytical prediction (see Eq. (31) (solid line - ) and from direct integration of Eq. (19) (solid line — ) for $t \in[11,17] s$ (zoomed area of Fig.(9))

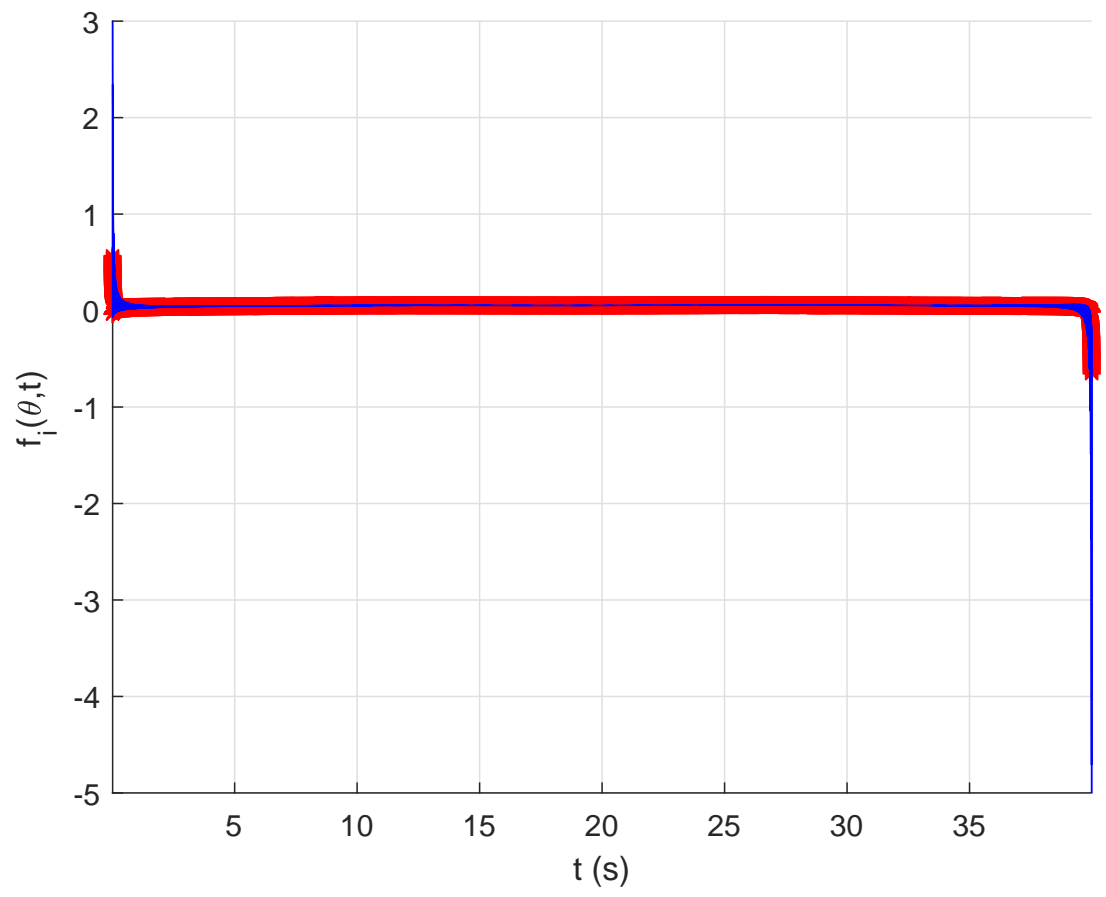

Fig. 11: Instantaneous frequency of $\theta(t)$ obtained from analytical prediction (see Eq. (31) (solid line - ) and from direct integration of Eq. (19) (solid line -) for $t \in[0,35] \mathrm{s}$

the one of the friction. Moreover, the oscillations of the pendulum in terms of angle are increased due to the inclusion of the effects of the support. These effects change the direction of the elongation from positive to negative (and vice versa) while ignoring the friction and the influence of the support, the elongation of the cable due to the oscillations does not change its sign. We spotted also that the pulling velocity for the system under given initial angle has a diverging effect (see Sect. (6)).

As an application of developed solutions, we considered a system of pulley which is encased in a space (for instance a tube). Via developed techniques, we show the possibility of impact of the system with surrounding walls and the possible time of the contact.

The developed method provides some tools for designing cable pendulums and their supports via taking into account the effect of velocity of the translation and the friction. 


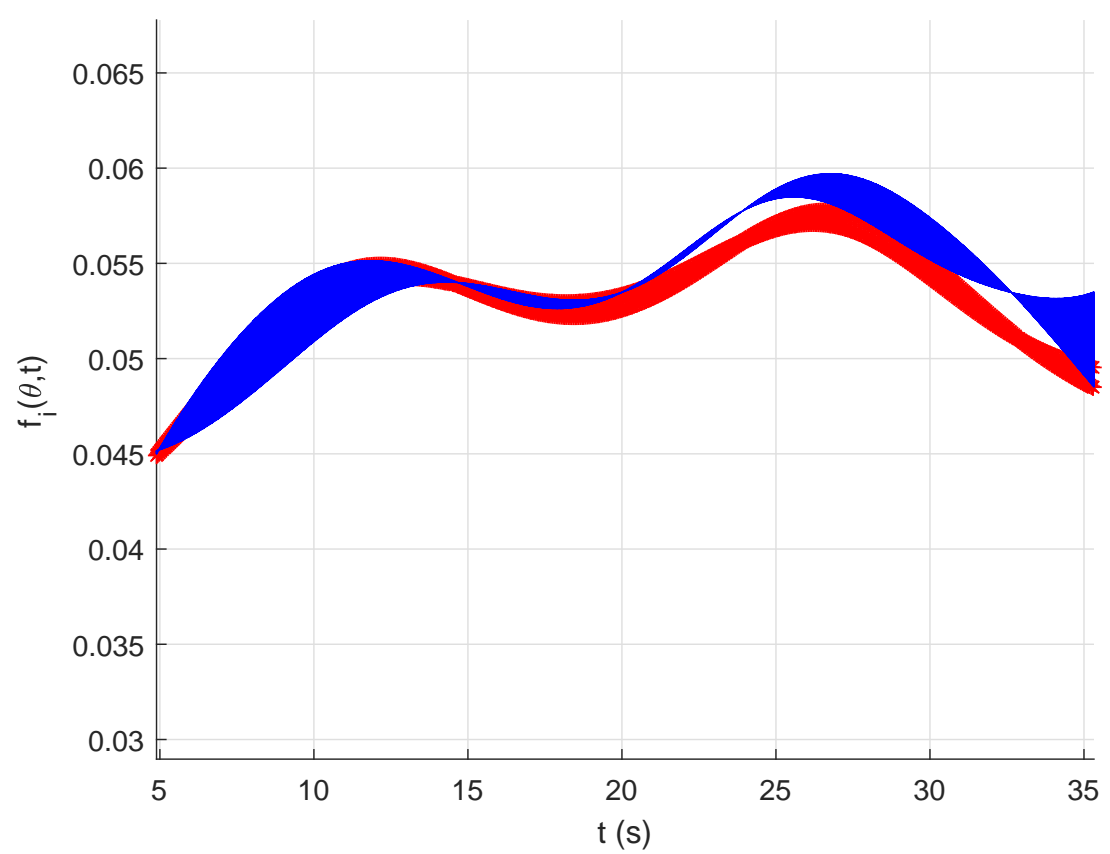

Fig. 12: Instantaneous frequency of $\theta(t)$ obtained from analytical prediction (see Eq. (31) (solid line - $)$ and from direct integration of Eq. (19) (solid line — ) for $t \in[5,35] s$ (zoomed area of Fig.(11))

\begin{tabular}{|l|r|r|r|r|r|r|r|r|r|r|}
\hline & $l_{0}$ & $g$ & $E S$ & $m$ & $T_{0}$ & $v_{0}$ & $R$ & $\mu$ & $\theta_{0}$ & $d$ \\
\hline Case (1) & 100 & 9.81 & 108 & 1000 & 8.4971 & 2 & 2 & 0.4 & 20 & 35 \\
\hline \hline Case (2) & 100 & 9.81 & 108 & 250 & 2.3045 & 2 & 2 & 0.4 & 20 & 35 \\
\hline
\end{tabular}

Table 3: System parameters for the two cases ; Units are the same as in Tab.(1) 


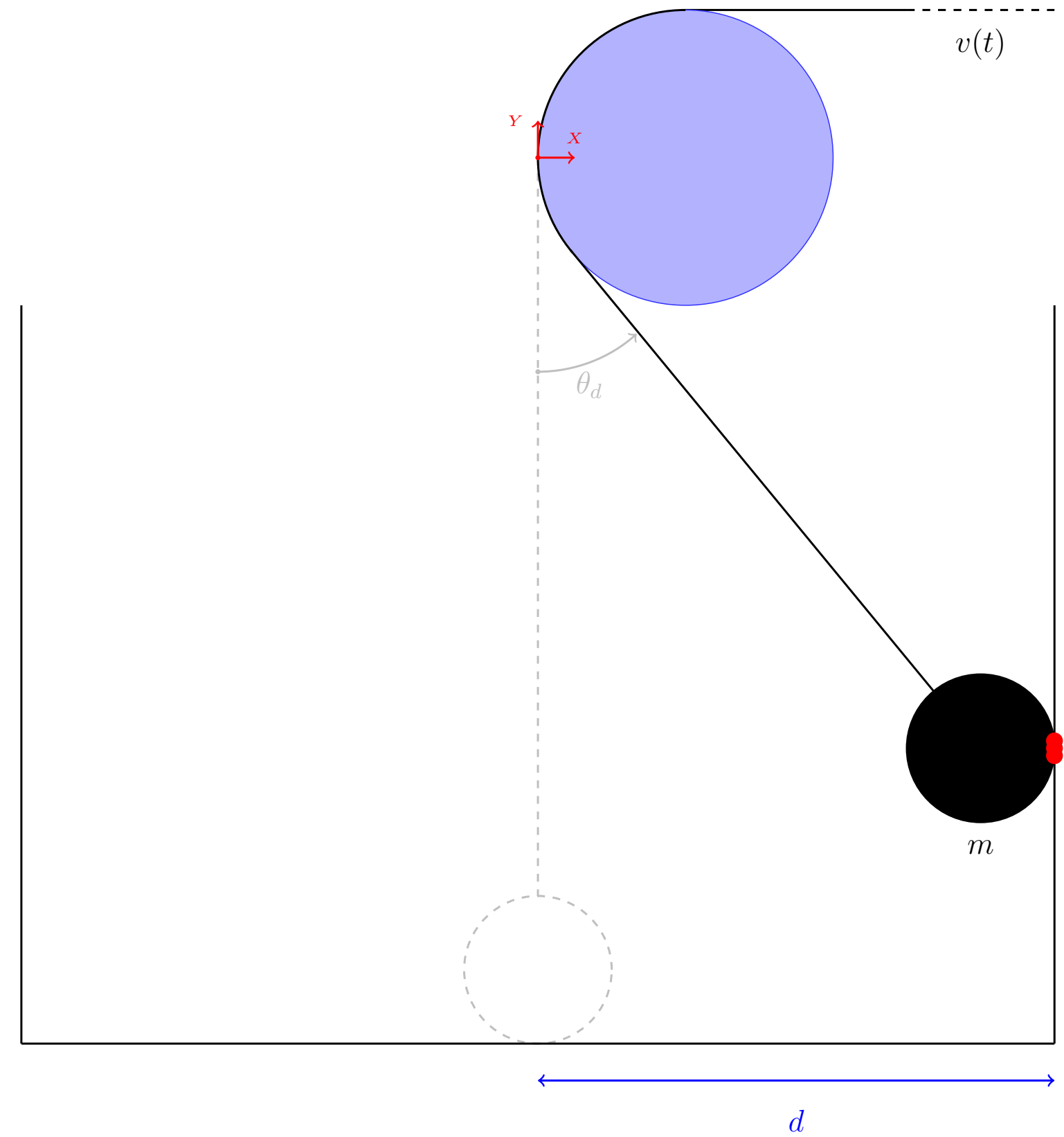

Fig. 13: The schematic of a drive pulley in an encased zone

\section{Appendices}

\section{A Expression of functions}

$$
\left\{\begin{aligned}
\Theta_{1,1}= & \theta_{0} \\
\Theta_{1,2}= & 0 \\
\Theta_{2,1}= & 0 \\
\Theta_{2,2}= & -\frac{7 v_{0} \theta_{0}}{8 \omega_{0} l_{0}} \\
\Theta_{3,1}= & \frac{1}{192} \theta_{0}\left(\frac{45 v_{0}^{2}}{l_{0}^{2} \omega_{0}^{2}}-\frac{\theta_{0}^{2}\left(2880 \omega_{0}^{6}-1012 \omega_{0}^{4}+137 \omega_{0}^{2}+11\right)}{\left(1-4 \omega_{0}^{2}\right)^{2}}\right) \\
& +\mu \frac{\theta_{0}^{2}\left(10 \omega_{0}^{2}-3\right)}{2 l_{0}\left(4 \omega_{0}^{2}-1\right)} T^{*}+\frac{\theta_{0}^{2}\left(22 \omega_{0}^{2}-7\right)}{6 l_{0}\left(4 \omega_{0}^{2}-1\right)} R^{*} \\
\Theta_{3,2}= & 0
\end{aligned}\right.
$$




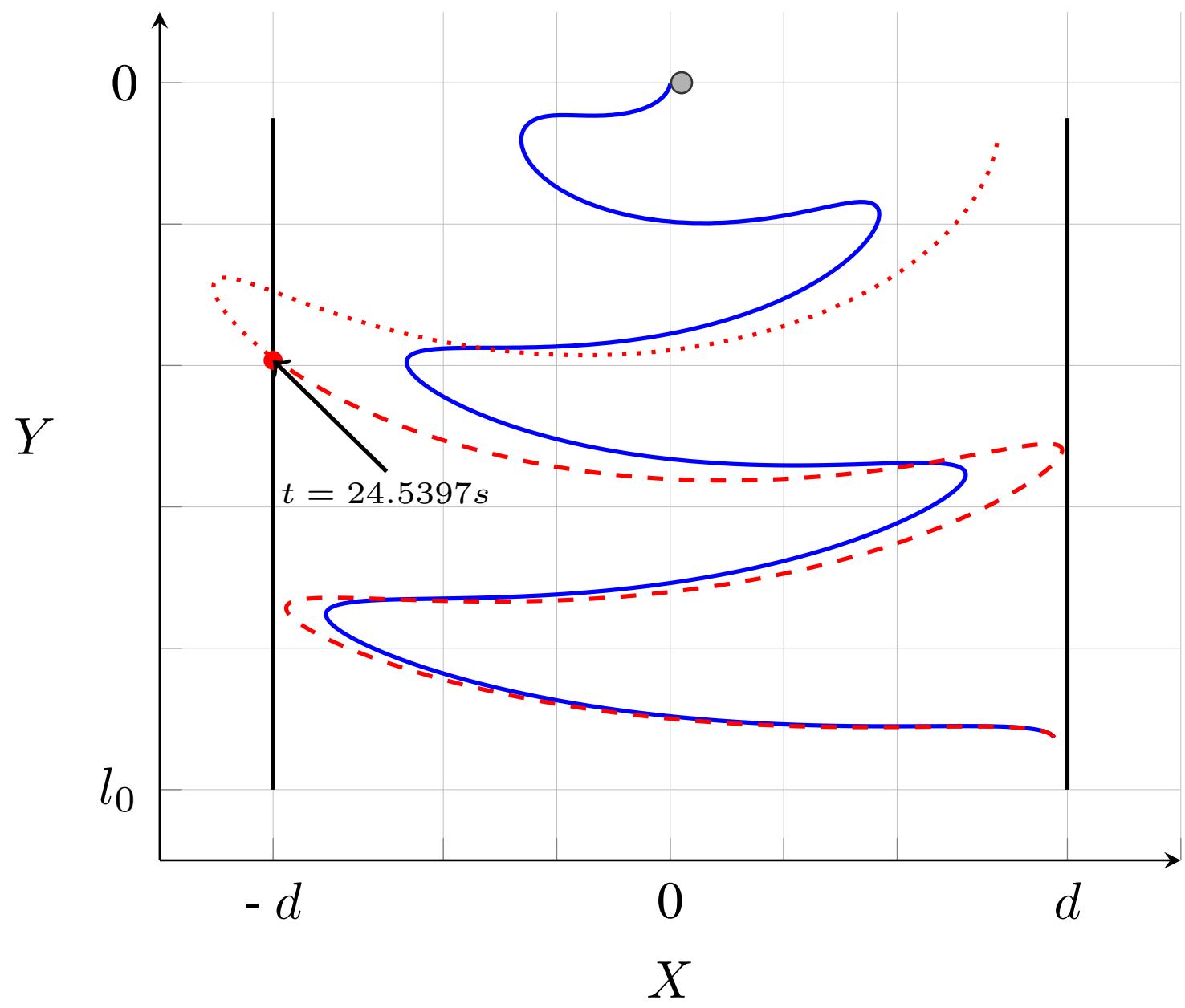

Fig. 14: Trajectories of the system within an encased zone for two cases:

Case (1) (solid line _ _); Case (2) Before impact:(dashed line - - -), After impact: (dotted line .....); Final position without impact ( $\mathrm{O}$ )

$$
\begin{aligned}
& \left\{\begin{array}{l}
\xi_{2,1}=-\frac{3 \omega_{0}^{4} \theta_{0}^{2} l_{0}}{4 \omega_{0}^{2}-1}+\mu \frac{\omega_{0}^{2} \theta_{0}}{\omega_{0}^{2}-1} T^{*}+\frac{\omega_{0}^{2} \theta_{0}}{\omega_{0}^{2}-1} R^{*} \\
\xi_{2,2}=0 \\
\xi_{2,3}=\frac{3 \theta_{0}^{2} l_{0} \omega_{0}^{2}\left(16 \omega_{0}^{2}-2 \omega_{0}-7\right)}{64 \omega_{0}^{2}-16}-R \theta_{0}-\mu \theta_{0} T^{*} \\
\xi_{3,1}=\mu^{2} \frac{\theta_{0}^{2} \omega_{0}^{2}}{1-4 \omega_{0}^{2}} T^{*} \\
\xi_{3,2}=-\frac{3 \theta_{0}^{2} v_{0} \omega_{0}^{4}\left(12 \omega_{0}^{2}-23\right)}{8\left(1-4 \omega_{0}^{2}\right)^{2}}-\mu \frac{\theta_{0} v_{0} \omega_{0}^{2}\left(\omega_{0}^{2}+7\right)}{8 l_{0}\left(\omega_{0}^{2}-1\right)^{2}} T^{*} \\
c_{1}\left(t_{0}\right)=\frac{\theta_{0}^{2} v_{0} \omega_{0}^{2}\left(\omega_{0}^{2}+23\right)}{8 l_{0}\left(\omega_{0}^{2}-1\right)^{2}} R^{*} \\
\left.\left.\xi_{3,3}=\mu^{2} \frac{\theta_{0}^{2}}{4} T^{*} \quad-9 \omega_{0}^{4} \cos \left(t_{0}\right)+5 \omega_{0}^{4}-7 \omega_{0}^{2}+2\right) \cos \left(\omega_{0} t_{0}\right)\right) \\
3 l_{0}\left(\omega_{0}-1\right)\left(\omega_{0}+1\right)\left(4 \omega_{0}^{2}-1\right) \\
+\frac{\theta_{0}^{2}\left(\left(2 \omega_{0}^{2}+1\right) \cos \left(2 \omega_{0} t_{0}\right)+3\right)}{6 l_{0}\left(\omega_{0}-1\right)\left(\omega_{0}+1\right)}+\frac{\theta_{0}^{2}\left(6\left(\omega_{0}^{2}-1\right) \omega_{0}^{3} \sin \left(t_{0}\right) \sin \left(\omega_{0} t_{0}\right)\right)}{3 l_{0}\left(\omega_{0}-1\right)\left(\omega_{0}+1\right)\left(4 \omega_{0}^{2}-1\right)} \\
c_{2}\left(t_{0}\right)=\frac{\theta_{0} \sin \left(\omega_{0} t_{0}\right)}{32 \omega_{0}\left(4 \omega_{0}^{2}-1\right) l_{0}^{2}}\left(2 \theta_{0}^{2} \omega_{0}^{2}\left(24 \omega_{0}^{4}-11 \omega_{0}^{2}-1\right) l_{0}^{2}+\left(15-60 \omega_{0}^{2}\right) v_{0}^{2}\right) \\
2 l_{0}^{2} \omega_{0} \sin \left(\omega_{0} t_{0}\right)
\end{array}\right. \\
&
\end{aligned}
$$




$$
c_{3}\left(t_{0}\right)=\frac{1}{8} \theta_{0} v_{0} \omega_{0}\left[\begin{array}{c}
\frac{2\left(R^{*}+\mu T^{*}\right)\left(\sin \left(\omega_{0} t_{0}\right)-\omega_{0} \sin \left(t_{0}\right)\right)}{l_{0}\left(\omega_{0}^{2}-1\right)} \\
-\frac{3 \theta_{0} \omega_{0}^{2}\left(\sin \left(2 \omega_{0} t_{0}\right)-2 \omega_{0} \sin \left(t_{0}\right)\right)}{4 \omega_{0}^{2}-1}
\end{array}\right]
$$

\section{Acknowledgments}

The authors thank following organizations for supporting this research:

(i) The "Ministère de la transition écologique et solidaire" and

(ii) LABEX CELYA (ANR-10-LABX-0060) of the "Université de Lyon" within the program "Investissement d'Avenir" (ANR-11IDEX-0007) operated by the French National Research Agency (ANR).

\section{Compliance with ethical statement}

The authors declare no conflict of interest. 


\section{References}

1. H. Max. Irvine. "Cable structures". Earthquake Engineering \& Structural Dynamics, 10(2):347-347, 1982.

2. F. Treyssede. "Free linear vibrations of cables under thermal stress". Journal of Sound and Vibration, 327(1-2):1-8, 2009.

3. G. Rega. "Nonlinear vibrations of suspended cables Part I: Modeling and analysis". Applied Mechanics Reviews, 2004.

4. A. Sofi. "Nonlinear in-plane vibrations of inclined cables carrying moving oscillators". Journal of Sound and Vibration, 332(7):1712-1724, 2013.

5. V. Gattulli, F. Di Fabio, and A. Luongo. "Simple and double Hopf bifurcations in aeroelastic oscillators with Tuned Mass Dampers". Journal of The Franklin Institute, 338(2-3):187-201, 2001.

6. R. Skutch. "Uber die bewegung eines gespannten fadens, welcher gezwungen ist, durch zwei feste punkte, mit einer constanten geshwindigkeit zu gehen, und zwischen denselben in transversal-schwingungen von geriger amplitude versetzt wird". Annalen der Physik une Chemie, 61, 1897.

7. A. Simpson. "On the oscillatory motions of translating elastic cables". Journal of sound and vibrations, 1972.

8. M.S. Triantafyllou. "The dynamics of translating cables". Journal of Sound and Vibration, 103(2):171-182, 1985.

9. N. V. Gaiko, A.W. Heemink, and W. T. van Horssen. "Transversal waves and vibrations in axially moving continua". 2017.

10. Anton O. Belyakov, A. P. Seyranian, and A. Luongo. "Dynamics of the pendulum with periodically varying length". Physica D, Elsevier , 238:1589-1597, 2009.

11. M. Babaz, L. Jezequel, C.-H. Lamarque, and P. Perrard. "Unusual expression of tension of a massless cable with application to the oscillations of a mass suspended to a cable with a variable length". Journal of Sound and Vibration, 363:446-459, 2016.

12. S.A. Bechtel, S. Vohra, K.I. Jacob, and C.D. Carlson. "The stretching and slipping of belts and fibers on pulleys". Journal of Applied Mechanics, 67:197-206, 2000.

13. H. Usabiaga, M. Ezkurra, M.A. Madoz, and J.M. Pagalday. "Experimental test for measuring the normal and tangential line contact pressure between wire rope and sheaves". Experimental techniques, pages 34-43, 2008.

14. C Westin and R. A. Irani. "Cable-Pulley interaction with dynamic wrap angle using the absolute nodal coordinate formulation". Proceedings of the fourth International Conference of Control, Dynamic Systems, and Robotics (CDSR'17), 2017.

15. A. H. Nayfeh and D.T. Mook. "Nonlinear Oscillations". Wiley-VCH Verlag GmbH, 1995.

16. V. Acary and B. Brogliato. "Numerical Methods for Nonsmooth Dynamical Systems". 2008.

17. N. Ben Brahim and Rousselet B. "Double scale analysis of periodic solutions of some non linear vibrating systems ". HAL, 2013.

18. C.-H. Lamarque, A. Ture Savadkoohi, E. Etcheverria, and Z Dimitrijevic. "Multi-scale dynamics of two couples nonsmooth systems". International Journal of Bifurcation and Chaos, 22:18 pages, 2011.

19. J.L. Meriam, L.G. Kraige, and J.N. Bolton. Engineering mechanics. Volume 1 : statics. 